\title{
Photic driving in the electro- encephalogram of children and adolescents: harmonic structure and relation to the resting state
}

\author{
V.V. Lazarev ${ }^{1}$, \\ D.M. Simpson ${ }^{2}$, \\ B.M. Schubsky and \\ L.C. deAzevedo ${ }^{1}$
}

\author{
'Laboratório de Neurofisiologia Clínica, Setor de Neurologia, \\ Instituto Fernandes Figueira, Fundação O swaldo Cruz, Rio de Janeiro, RJ, Brasil \\ 2Programa de Engenharia Biomédica (COPPE), Centro de Tecnologia, \\ Universidade Federal do Rio de Janeiro, Rio de Janeiro, RJ, Brasil
}

\section{Correspondence \\ V.V. Lazarev \\ Laboratório de Neurofisiologia \\ Clínica \\ Instituto Fernandes Figueira \\ FIOCRUZ \\ Av. Rui Barbosa, 716 \\ 22250-020 Rio de Janeiro, RJ \\ Brasil \\ Fax: +55-21-2553-8094 \\ E-mail: vlad.v@ iname.com or \\ vlazarev@ hotmail.com}

Research supported by CNPq and FIOCRUZ (No. 300567/1994-4).

Received November 22, 2000 Accepted September 3, 2001

\section{Abstract}

In order to identify latent bioelectrical oscillators, 15 normal subjects (aged 9-17 years, 8 males, 7 females) were subjected to intermittent photic stimulation. The EEG amplitude spectra corresponding to the 11 fixed frequencies of stimulation presented (3-24 Hz) were combined to form "profiles" of the driving reaction in the right occipital area. The driving response varied with frequency, and was demonstrable in $70-100 \%$ of cases (using as criterion peak amplitudes $20 \%$ larger than those of the neighbors). The strongest responses were observed at the frequency closest to the alpha peak of the resting EEG. A secondary profile maximum was in the theta band. In 10 subjects, this maximum exceeded half the alpha peak (with an average of $72.4 \%$ of the alpha peak), while in the resting spectra, theta amplitudes were much lower than the alpha maxima. This responsiveness in theta activity seems to be characteristic of prepubertal and pubertal subjects. The profiles and resting EEG spectra showed a highly significant Pearson's correlation, with the peak in the theta band of the profiles being the main difference observed between them. The correlation coefficient was significantly correlated with the ratio of the maxima in the theta and alpha bands $(\mathrm{R}=-0.77, \mathrm{P}<0.001)$. The correlation coefficient between profile and resting spectrum may be a useful indicator in screening methods used to reveal latent cerebral oscillators. Profiles for the second and third harmonics were correlated with those of the first harmonic (fundamental frequency), when considering the corresponding EEG frequencies. Peak frequencies in all three profiles were close to those of the individual's background alpha rhythm, and peak amplitudes in higher harmonics were not much lower than those of the fundamental frequency (mean values of 84 and $63 \%$, for second and third harmonics, respectively).

\section{Key words}

- Electroencephalogram

- Intermittent photic

stimulation

- Photic driving

- Harmonic

- Children

- Adolescents 


\section{Introduction}

In electroencephalography, the activation procedures or functional tests enhance the manifestation of latent neurophysiological mechanisms or pre-existing abnormalities, and may induce abnormal findings in an otherwise normal electroencephalogram (EEG) (1). Their importance in clinical work has increased due to the heightened discriminating power of the computerized quantitative methods and functional brain mapping that are presently being applied to the analysis of the EEG. Intermittent photic stimulation (IPS) is one of the most important functional tests during neurophysiological examinations. It can induce in the EEG photic driving - a physiologic response consisting of rhythmic activity time-locked to the stimulus at a frequency identical or harmonically related to that of the stimulus (2). In the EEG spectrogram, driving appears as sharp amplitude peaks at these frequencies.

The hypothesis of the resonance nature of the driving response was suggested as early as the nineteen forties (3) and later was accepted and experimentally confirmed by many investigators (4-10). On this basis, IPS has been used to test some basic neurophysiological mechanisms taking into account modern concepts of the organizing role of the bioelectrical rhythms in the brain $(11,12)$. It is currently believed that the differentiated working of different neural circuits within the brain occurs at different specific frequencies, and the principle of resonance filtering of stimuli underlies the reactions to external stimulation and coding of information $(4,7)$. The resonance properties of the EEG oscillators can be enhanced by IPS. A gradual continuous change in frequency of stimulation across the entire EEG frequency range has allowed an individual's profile of reactivity to be calculated, based on the set of peaks elicited in the EEG spectrogram. This profile apparently reflects the characteristic EEG oscillators in different bands
$(6,8)$. These oscillators were found to be very variable across a group of subjects, but in each individual, the frequencies of the peaks proved to be very stable over a long period of time (6). Other investigators also observed large interindividual variability and long-term individual stability of the driving reactions (4). Significantly, however, the resonance driving peaks not only coincided with the background peaks, such as those of alpha activity, but also appeared at the frequencies where no peaks were observed in the background EEG $(6,8)$. This potential for revealing the individual latent oscillators makes the IPS test very valuable and promising in both clinical and basic neurophysiological studies and permits obtaining additional information not present in the resting EEG (1,13-16).

Photic driving can directly influence psychophysiological processes. The presence of such response in the premotor, motor and sensorimotor cortical areas may indicate a low resistance to interference from stimulation in an individual's motor activity (17). Driving at the alpha frequencies can produce a relaxing effect, and pronounced physiological reactions to the IPS may be observed in heart rate, breathing, galvanic skin resistance $(18,19)$, as well as in regional cerebral blood flow (20). These effects have been utilized in some forms of therapy (18).

The application of IPS to normal subjects permits a more detailed study of the EEG typology and the EEG correlates with individual psychophysiological characteristics (21). In particular, it has been suggested for use in the selection of candidates for space flight (17). The parameters of the driving response may serve as sensitive EEG indicators of the peculiarities of age and brain maturation $(1,16,22)$, as well as of gender differences (13).

In clinical practice, IPS is part of most routine examinations. Its utilization is very often limited to simply ascertaining the presence of a photoparoxysmal response in so- 
called photosensitive epilepsy $(23,24)$. This abnormal response can only be observed in $5-10 \%$ of the epileptic population (25), predominantly in generalized epilepsies, and is not typical of focal epilepsies (26). Application of IPS can, however, also be effective in the study of epilepsy without a convulsive response. It was shown through an increase in regional cerebral blood flow that the epileptogenic foci are very sensitive to certain frequencies of photic stimulation, which provoke local physiological changes, even in the absence of paroxysmal manifestations (27).

For non-paroxysmal responses to IPS, many clinical correlates are described in the literature. They may serve as additional EEG signs of cerebral pathology, not always present in the resting EEG. For example, exaggerated driving at low flickering frequencies usually signifies acute or subacute neuronal dysfunction (28). Cortical lesions of a destructive type may cause ipsilateral depression or attenuation of driving, whereas irritative lesions, such as those of epileptic scars, may lead to an increased response on the side of a focus $(29,30)$. A locally increased rhythmic response in delta or beta frequencies may indicate a focal disturbance, and a reduced or disproportionately hypersynchronized response within the alpha band may reflect certain functional alterations (24). However, a generalized low voltage or absence of photic driving is of little diagnostic significance because some normal individuals are not responsive to IPS (28).

In neurology, the driving response to high frequency IPS is considered by some authors as the only reliable EEG indicator of headache (15,31). In psychiatry, a decreased driving response at the alpha frequencies has been found in schizophrenia (10). In these patients alpha driving may be higher in the frontal areas than in the occipital region (32), probably indicating some signs of "hypofrontality". EEG photic driving is also utilized in the EEG evaluation of drug ef- fects and in predicting the efficacy of therapy (33), in certain cases being more sensitive than the resting EEG (34).

Expressive EEG driving responses are very often recorded not only at the frequency of IPS (to be called the fundamental or first harmonic) but also at higher harmonics (integer multiples of the fundamental frequency) (3,35-37). In experiments with animals and humans, it was shown that such harmonics are not just an artificial result of Fast Fourier Transform, but reflect real physiological reactions (36). However, very few authors pay attention to the harmonic composition of the EEG driving response. Some still consider its significance as poorly understood (1). Nevertheless, there are some data showing a certain diagnostic potential of this aspect of the reaction. The ratio of harmonic amplitudes proved to be a fine indicator of the functional states in normal subjects $(5,35)$. It may depend upon the clinical state of the patients, as found in renal disease (38). The gender EEG differences in older ages were found only in the second harmonic of the driving response (13). In geriatric clinical work, only decreased harmonic driving differentiated the patients suffering from early dementia from age-matched depressed ones, whereas neither resting EEG nor driving reaction at the frequency of stimulation showed any differences (14). Certain alterations were found in the dynamics of the second harmonic during high frequency IPS in migraine (39).

In spite of the great interest in EEG photic driving, the peculiarities of the responses in children and adolescents have not received great attention, especially using quantitative EEG methods. The responses of normal subjects in this age group need to be described further to permit more effective application of IPS to children in neurological and psychiatric studies.

The objectives of the present research were to propose and test a simple methodology for the detailed quantitative description 
of the driving reaction using standard equipment available in most clinical EEG laboratories, which could readily be utilized in routine examination of patients. The main thrust of this approach is to combine the amplitudes of the EEG reactions to IPS at different fixed frequencies in a single graph, equivalent to an amplitude spectrum. This methodology is aimed at revealing any additional potential EEG oscillators not present in the resting EEG of children and adolescents. An analysis of the so far insufficiently explored harmonic content of the driving reactions is also presented.

\section{Material and Methods}

The volunteers were 15 children and adolescents, 8 males and 7 females, with a median age of 13.3 years (range 9 to 17 years). None had a history of neurological, psychiatric or drug-related illness and none was overtly left handed. The study was approved by the local Ethics Committee. Written informed consent was obtained from the participants and their parents.

EEG signals were recorded during a state of relaxed wakefulness (initial background, 2-3-min duration), during IPS at frequencies of $3,4,5,6,8,10,12,15,18,21$ and $24 \mathrm{~Hz}$, and during relaxed wakefulness between stimulations. The subjects kept their eyes closed throughout the experiment. Each stimulation consisted of flashes presented at a fixed frequency for 20-30 s, with the same periods between stimulation runs. In 12 of the 15 subjects, this series was repeated. Considerations of comfort and therefore cooperation of the subjects did not allow more extended stimulation periods. The photostimulator was a xenon lamp whose flash of approximately white light had a duration of less than $20 \mu \mathrm{s}$. The lamp was positioned at a distance of $25 \mathrm{~cm}$ from the eyes, with dim surrounding light.

The EEG was recorded on an 18 channel Nihon Kohden polygraph (EEG-4418), with monopolar leads at 16 scalp points according to the International 10/20 System, with references at the ipsilateral earlobes. The recording characteristics were: $0.3 \mathrm{~s}$ time constant, $70 \mathrm{~Hz}$ low-pass filter and $15 \mu \mathrm{V} /$ $\mathrm{mm}$ sensitivity. EEG signals were simultaneously recorded on paper and magnetic tape (tape recorder TEAK XR-7000), and then digitized by the computerized BrainScan (Neurotron) system. The digital sampling frequency was $256 \mathrm{~Hz}$. The EEG was not digitized during at least $5 \mathrm{~s}$ after each stimulation in order to exclude a possible short post-stimulation effect (9).

The present study was restricted to the right occipital region, generally considered as representative for the study of visual stimulation $(3,20,37)$. The results of spectral analysis (using the Fast Fourier Transform) were presented in the form of amplitude spectra, i.e., the square root of the power spectrum, with a frequency resolution of $0.5 \mathrm{~Hz}$. Since in the present study EEG spectra with and without stimulation are compared, a spectral resolution was selected that was considered suitable for the study of background EEG activity, while also clearly showing the response to photic stimulation. The presence of the driving response at the frequency of stimulation (the fundamental or first harmonic) and its higher (i.e., second and third) harmonics was ascertained with in-house software, using as a criterion for the detection of the response an amplitude peak which was at least $20 \%$ higher than the amplitudes at the adjacent frequencies in the range \pm 1 Hz. Computer simulation studies showed that this criterion gave a false-positive rate of less than $5 \%$ when the spectrum of signals is white, i.e., when there is no response to the stimuli. This approach will be referred to as the " $20 \%$ criterion".

In order to compare the responses to all the frequencies of stimulation, amplitude spectra were estimated for the EEG signals during each stimulation run, and combined in the "individual frequency profile of the 
driving reaction" (or "profile") by extracting from each spectrum only the component obtained at the stimulus frequency. Similar profiles were constructed for the second and third harmonics of the stimulus frequency.

The similarity between these different profiles was evaluated by Pearson's correlation coefficient $(\mathrm{R})$. Statistical significance of changes in profile from the first to the second series of stimulation was evaluated by the Wilcoxon test. In the profiles the abscissa was plotted according to the frequency of stimulation (Figure 1A) or according to the frequency of the EEG (Figure 1B). For the fundamental frequencies the two scales are therefore equal, but for the second harmonic, the latter show values twice as high as those of the former.

\section{Results}

Some of the individual spectrograms of the EEG with the driving responses to IPS are presented in Figure 2 with an example of a particularly strong reaction to IPS at $3 \mathrm{~Hz}$, especially at the higher harmonics (Figure 2A).

In the right occipital area $(\mathrm{O} 2)$, such responses (according to the $20 \%$ criterion) in each of the two first harmonics took place during different stimulation frequencies in $67-100 \%$ of the subjects over the 11 stimulus frequencies, with the maximum for the first harmonic at 4, 15 (93\% in both) and $24 \mathrm{~Hz}$ (100\%) (Figure 3). In one subject (SV, a 13year-old boy), in strong contrast to the others, the driving effect in the first harmonic was observed only during the $24-\mathrm{Hz}$ stimulation, and in the second one during the 3-, 8-, 10- and 12-Hz stimulations. Therefore, most of the group data below are presented for 14 subjects, excluding SV. In these 14 subjects, the driving reaction was detected in the first harmonic at, on average, $9.7 \pm 1.15$ (mean $\pm \mathrm{SD}$ ) frequencies or $88 \%$ of the 11 frequencies presented. In the second harmonic, the corresponding results were $8.8 \pm$
2.3 or $80 \%$ of frequencies, and in the third harmonic $6.4 \pm 2.1$ or $58 \%$ of the stimulations.

Two examples of the individual frequency profile for the first harmonic of the driving reaction are shown in Figure 1A and refer to
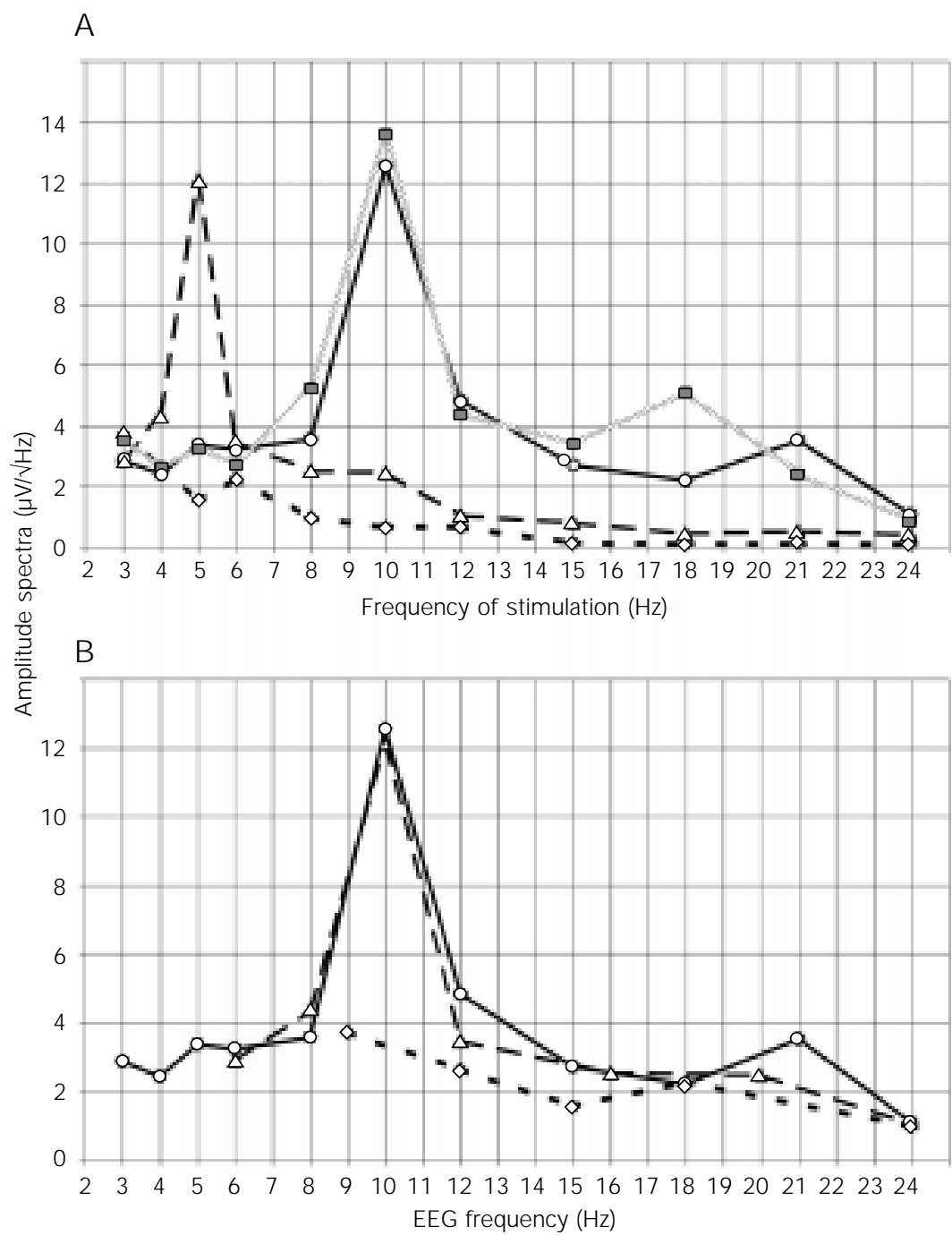

Profile 1 Profile 2

O 1 harm $\triangle 2$ harm $\diamond 3$ harm $\square 1$ harm

Figure 1. An example of individual driving profiles (subject TG), constructed by combining the amplitude spectra (in $\mu \mathrm{V} / \mathrm{VHz}^{1}$ ) obtained during stimulation at different frequencies. $\mathrm{A}$, Profiles for the first (Profile 1 ) and second (Profile 2) series of intermittent photic stimulation (IPS). For the first series, the profiles for the first three harmonics (1, 2 and 3 harm) are given. For the second series, only that of the fundamental one is shown. The abscissa shows the frequency of stimulation, in $\mathrm{Hz}$. B, Profiles for the first three harmonics of the first IPS series (as used in Figure 1A), but with the abscissa showing the EEG frequency, in $\mathrm{Hz}$. 

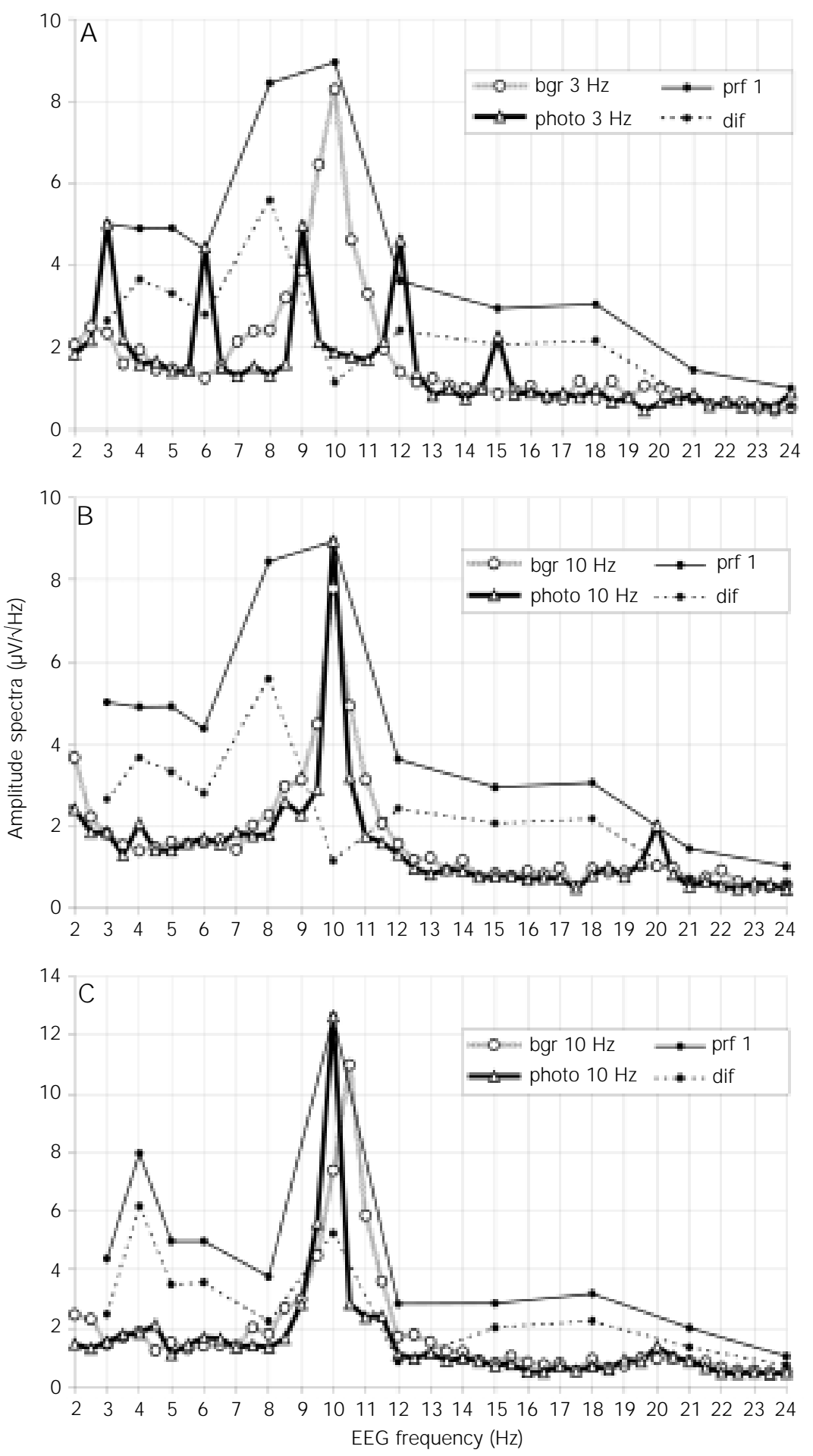

Figure 2. EEG spectra for individual subjects in the first series of intermittent photic stimulation (IPS), in the resting state (bgr) and during IPS (photo), together with the driving profiles (prf 1) and "difference" profiles (dif), showing an increase in the amplitude spectra at each frequency of IPS in relation to the background. A, Subject MA, and IPS of $3 \mathrm{~Hz}$ (for spectrum). B, Subject MA, and IPS of $10 \mathrm{~Hz}$ (for spectrum). C, Subject TI, and IPS of $10 \mathrm{~Hz}$ (for spectrum). The abscissae show the EEG frequency. the first and second series of stimulation in subject TG. These show the typical shape, with a maximum at the frequency of $10 \mathrm{~Hz}$. The two profiles proved to be very similar $(\mathrm{R}$ $=0.94, \mathrm{P}<0.001)$. In 11 subjects who passed through two series of stimulation (excluding $\mathrm{SV})$, the average $\mathrm{R}$ was $0.91 \pm 0.08(\mathrm{P}<0.001)$. A significant difference $(\mathrm{P}<0.05$, Wilcoxon test) between the profiles from the first and second series of stimulation was found in this group only at $12 \mathrm{~Hz}$ (showing a decrease) and at $18 \mathrm{~Hz}$ (increase).

For all 14 subjects (excluding SV) the maximum of the profile was in the first harmonic in the alpha band: 10 of them at 10 $\mathrm{Hz}, 3$ at $8 \mathrm{~Hz}$ and 1 at $12 \mathrm{~Hz}$. In the 12 subjects who showed a driving reaction at these frequencies according to the $20 \%$ criterion, this maximum had an average value of $11.33 \pm 3.14 \mu \mathrm{V} / \mathrm{JHz}^{1}$ while the average amplitude in the background alpha peak was $6.97 \pm 2.21 \mu \mathrm{V} / \sqrt{ } \mathrm{Hz}$. The background peak frequency differed from that of the profile by no more than $0.5 \mathrm{~Hz}$.

In 6 subjects there was an additional peak in the profile in the theta band $(4-6 \mathrm{~Hz})$, with an average amplitude of $7.72 \pm 2.92 \mu \mathrm{V} /$ $\sqrt{ } \mathrm{Hz}$. This was $76 \%$ of the profile alpha peak amplitude (Figure 4A). Only one of these subjects had a prominent theta peak in the spectra of the initial background EEG (Figure 4D: PO). In another 4 subjects without a prominent theta peak in the profile, the profile maximum in the theta band had an average amplitude of $7.50 \pm 2.70 \mu \mathrm{V} / \sqrt{\mathrm{Hz}}$ or $67 \%$ of the alpha peak (Figure 4B). In contrast, in the initial background of 13 subjects, the

${ }^{1}$ The unit of the power spectral density is $\mu \mathrm{V}^{2} / \mathrm{Hz}$, as normally used for the background EEG, and therefore that of the amplitude spectrum is $\mu \mathrm{V} / \mathrm{VHz}$. This will be used throughout the current paper. In the driving reaction, consisting of sinusoidal oscillations at a number of well-defined frequencies, the amplitudes of the harmonics (in $\mu \mathrm{V}$ ) can be calculated by multiplying the amplitude spectral values at the corresponding frequencies by 1.41 (for the spectral resolution of $0.5 \mathrm{~Hz}$ employed). 


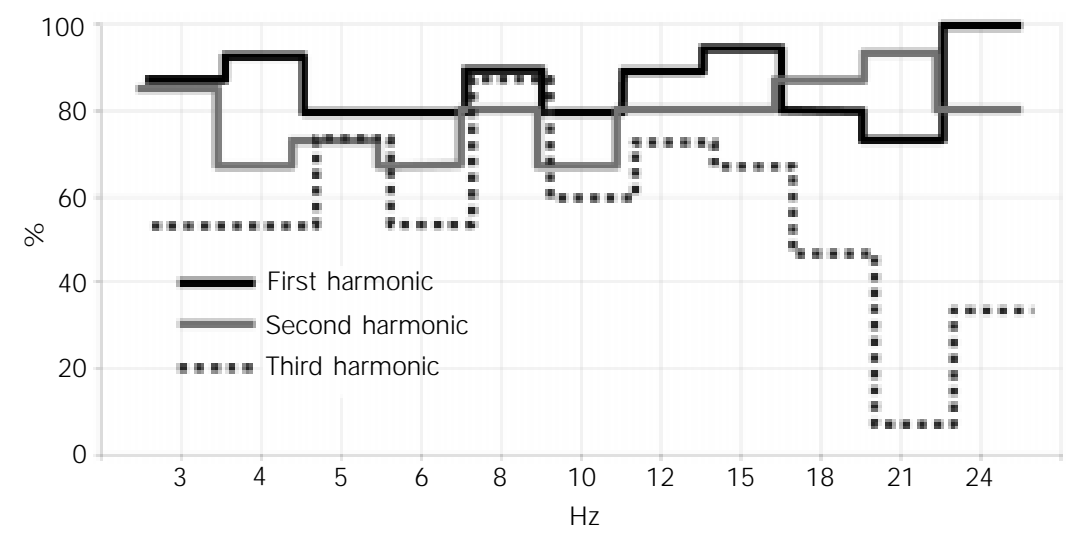

Figure 3. Percentage of subjects showing a driving response (according to the criterion of peak values $20 \%$ larger than those of adjacent frequencies) in the first three hamonics of each frequency of the first series of intermittent photic stimulation. A hundred percent indicates a driving response in all 15 subjects of the group studied.
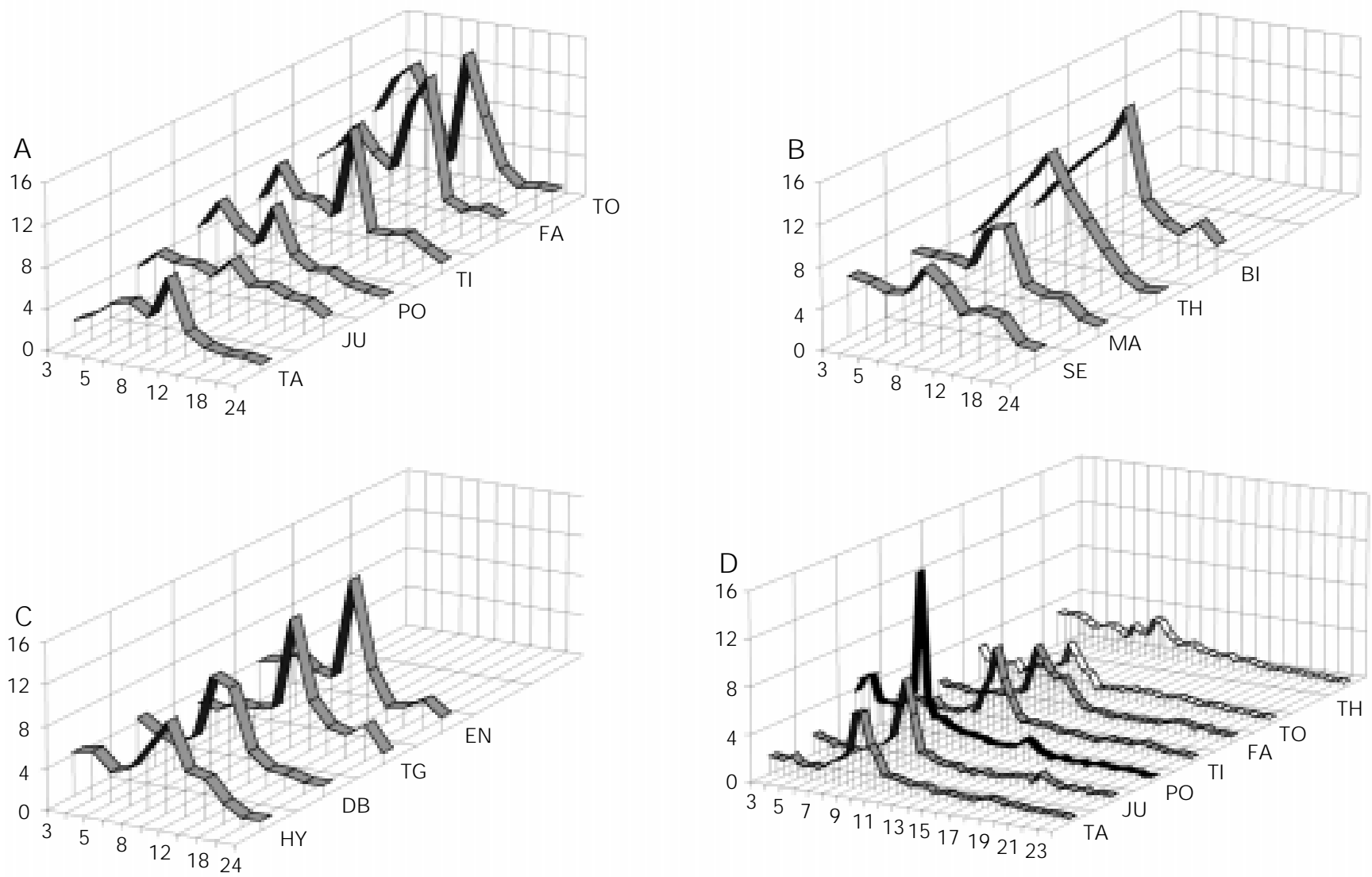

Figure 4. Individual driving profiles (first harmonic in the first series of intermittent photic stimulation) classified according to the reaction in the theta band (see Figure 1). The abscissae show the frequency of the EEG and stimulation, in $\mathrm{Hz}$; the ordinates show amplitude spectra, in $\mu \mathrm{v} / \mathrm{VHz}$. TA, J U, PO, etc., indicate the subjects. A, Six subjects with theta peak amplitudes exceeding half their maximum in the alpha band. B, Four subjects without theta peak, but with amplitude maxima in the theta band exceeding half their maximum in the alpha band. $\mathrm{C}$, Four subjects with amplitude maxima in the theta band less than half their maximum in the alpha band. D, Initial background EEG spectra of subjects in group A, and one from group B (TH - who did not show a dominating alpha peak in the resting EEG). 
ratio of the theta maximum to the alpha peak was $0.29 \pm 0.09$, with a mean theta amplitude of $2.35 \pm 1.15 \mu \mathrm{V} / \sqrt{ } \mathrm{Hz}$, and only in 1 subject were these maxima comparable (ratio of 0.81 ; Figure 4D: TH).

In the beta band, the maximum of the profiles was generally lower than that of the theta band, reaching $33 \pm 15 \%$ of the peak value in the alpha band.

The average correlation coefficient between the driving profile and the initial background EEG spectra was $\mathrm{R}_{\mathrm{db}}=0.82 \pm 0.13$ $(\mathrm{P}<0.001)$. In the whole group (except for subject $\mathrm{PO}$ for whom the background theta peak exceeded that of alpha measured at the frequencies of stimulation), the values of $R_{d b}$
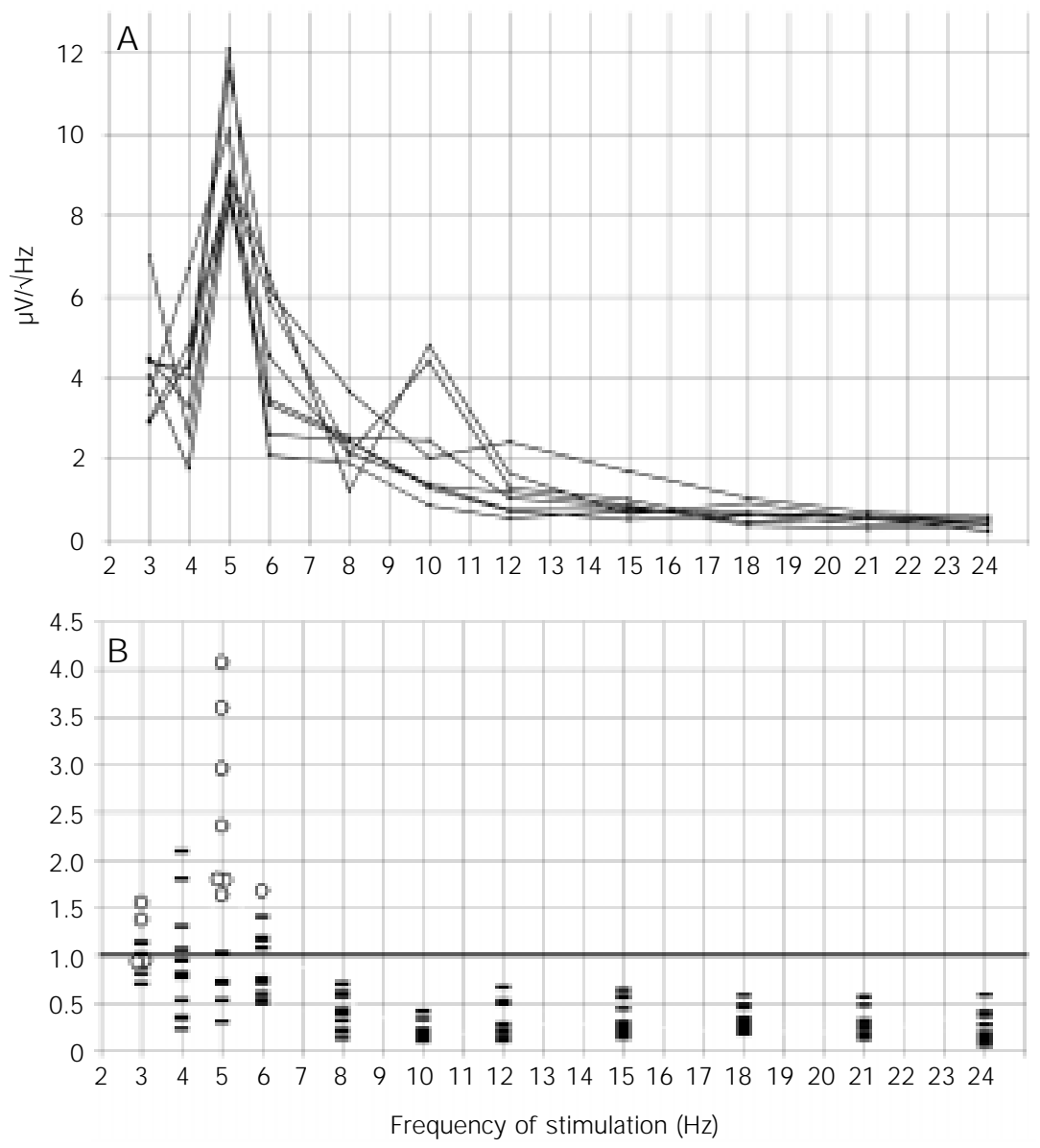

Figure 5. Driving profiles for second harmonic. A, Superposition of profiles for 8 subjects who had a profile maximum during intermittent photic stimulation at $5 \mathrm{~Hz}$ (see Figure 1). B, Superposition of amplitude ratios "second harmonic/first harmonic" in 12 subjects. Circles: maximum of the ratios in each subject. The abscissae show the frequency of stimulation. between the profile and the initial background were negatively correlated with the amplitude of the profile theta maximum $(\mathrm{R}=$ $-0.61, \mathrm{P}<0.01)$ and with the ratio of theta to alpha maxima $(\mathrm{R}=-0.77, \mathrm{P}<0.001)$.

The difference between the profile values and the background amplitude spectra at the same frequencies was almost always positive, indicating increased amplitude spectra during stimulation. The maximum of such an increase was, on average, $6.87 \pm 3.37 \mu \mathrm{V}$ / $\sqrt{\mathrm{Hz}}$. In 4 subjects this maximum was observed at $4 \mathrm{~Hz}$, in 1 subject at $15 \mathrm{~Hz}$, and in the remaining 10 subjects in the alpha band.

The driving profile constructed from the second harmonics had its maximum at an IPS of 3-6 Hz, with peaks at $5 \mathrm{~Hz}$ in 8 subjects (Figures 1A and 5A). In 12 subjects with a driving effect at these frequencies (according to the $20 \%$ criterion), the maximum ratio of the amplitude of the second harmonic to that of the first harmonic measured at the same frequency of stimulation was, on average, $2.06 \pm 1.0$, and exceeded 1.0 in 10 cases (Figure 5B).

Considering the frequency of the EEG rather than that of stimulation, the maximum of the profile of the second harmonic fell in the alpha band (Figure 1B). In all cases except one, it exactly coincided with the strongest response found for the profile of the fundamental frequency, and therefore, as stated above, was close to the peak alpha frequency of the background. The maximum amplitude of the profile for the second harmonic was on average $0.84 \pm 0.29$ of that for the first, and this ratio exceeded 1.0 in 4 cases.

In 13 subjects, the average correlation coefficient $\mathrm{R}$ was $0.9 \pm 0.08(\mathrm{P}<0.001)$ between the profiles of the first and second harmonics, comparing the profiles on the basis of the frequencies of the EEG rather than those of the stimuli. In one subject in whom the peak frequencies did not coincide, $\mathrm{R}$ was $0.55(\mathrm{P}<0.05)$. 
In the profiles of the third harmonic, the maximum was observed at stimulation frequencies of 3 or $4 \mathrm{~Hz}$. The ratio of the profile maximum of the third harmonic to the amplitude of the first at the same frequency of stimulation was, on average, $1.18 \pm 0.55$ (Figure 1A), exceeding 1.0 in 5 cases, and its ratio to the maximum of the first harmonic was $0.63 \pm 0.30$ (Figure 1B).

\section{Discussion}

The results demonstrate very pronounced photic driving responses in almost all of the children and adolescents examined. In general, the reactivity seemed to be rather stronger than that observed in adults, according to the literature $(1,30)$.

The similarity of the profiles of the first and second series of stimulation in the same subjects is indicative of the ability of the profile to describe certain relatively stable characteristics of the reactivity. This has been considered to reflect constitutional features of the EEG $(4,6)$. On the other hand, some authors have found variable components of the reaction in photic driving, which are determined by the current functional state $(5,35,37)$. Such components are probably reflected in the differences between the driving profiles of the two series of stimulation, such as the significant amplitude increase at $18 \mathrm{~Hz}$ and the decrease at $12 \mathrm{~Hz}$. These could be caused by tiredness, orienting reaction or influence from some uncontrolled mental processes, which might also contribute to somewhat lower values of the between-series correlation in some subjects.

A high correlation between the driving profile and the background EEG spectra may be considered evidence of the above-mentioned concept of the resonance mechanisms of the driving response (4-10). This is most clearly illustrated by the maximum increase in amplitude occurring during stimulation near the peak alpha frequencies of the background EEG $(3,5,35)$. In this case, the peak in the EEG spectra shifts towards the frequency of stimulation and there is a sharp decrease in amplitude at the frequency of the background alpha peak (see Figure 2C). However, when the frequency of stimulation coincides exactly with the peak alpha frequency, the amplitude increase in relation to the background may be reduced (see Figure $2 \mathrm{~B}$ ), and thus does not clearly reflect the strength of the driving reaction. Other authors also observed that the largest amplitude increase took place in individuals with low-amplitude resting EEG (19). There probably are limits to the interneuronal synchronization at a given frequency, which are almost reached in the high-amplitude alpha rhythm. However, it should be taken into account that the driving response may be evident as a clear spectral peak, even without an increase in the spectrum at the stimulation frequency, or its harmonics, when there is a pronounced decrease at the adjacent frequencies. Such a decrease is the manifestation of the orienting component of the reaction to IPS normal for any stimulus (37).

An enhanced driving reaction in the theta band in the absence of a background theta peak demonstrates the most important virtue of the IPS as a functional test, i.e., its ability to reveal and emphasize resonance of the latent neuronal oscillators and interneuronal sources of synchronization (1,13-16). This activation in the theta band relative to the background was comparable to, or even larger than that in the alpha band. The predisposition to resonance responses of theta oscillations and the responsiveness at the lower frequencies may be a functional characteristic of the prepubertal and pubertal periods. There is no evidence in the literature on the driving reaction that heightened theta responsiveness is found in normal adults. A psychophysiological study including adult series is planned in order to verify and reveal the peculiarities and individual features of the driving reactions of the developing and maturing brain $(16,22)$. The results obtained 
also show that the reduced values of the correlation coefficient between the driving profile and the background spectra may be useful in screening methods to reveal latent cerebral oscillators, including pathological forms of bioelectrical activity.

The driving responses observed in the EEG at twice or three times the frequency of stimulation, i.e., at the second and third harmonics, were strongly pronounced. These were found to be almost as common as the reaction at the fundamental frequency. The maxima of the profiles of the second and third harmonics occurred at the low stimulation frequencies of 3-6 Hz, and the peaks were appreciably higher than those obtained at the fundamental frequency. These maxima fell at EEG frequencies close to the background alpha peak and coincided with the maximum of the response at the fundamental frequency, showing only slightly decreased amplitudes compared to it. The profiles of different harmonics plotted according to the frequency of their effects evoked in the EEG (see Figure 1B) proved to be very similar in form and highly correlated. This is in accordance with the above mentioned concept of the resonance mechanisms of the photic driving (4-10) and demonstrates that the most powerful alpha oscillators appear to manifest their predominant resonant behavior even when stimulated at half or one third of the subject's alpha frequency (40). This should be taken into account when applying continuously changing frequencies of stimulation $(6,8)$ when the spectral power at a given frequency must contain a contribution from the stimuli at half, one third, etc., of this frequency. As a result, the EEG spectra are biased: the lower frequencies represent the driving effects of only the stimulation at low frequencies, whereas higher frequencies receive additional contributions from harmonics, and thus are equivalent to a longer duration of stimulation. Continuously varying stimulus frequencies have the obvious virtue of allowing a better match be- tween the frequencies of stimulation and those of latent neural oscillators than is possible with fixed stimulus frequencies (which are most common and routinely available in EEG examinations). However, the results obtained here suggest that exact coincidence of the frequencies of the stimulus and brain oscillator is not always required for an expressive driving effect. Moreover, a slight frequency difference between them may produce an enhanced increase in driving amplitude.

The resonance effects of the driving response are not fully understood. While, for example, the manifestation in the EEG of the second harmonic during stimulation at $5 \mathrm{~Hz}$ is similar to that of the first harmonic at 10 $\mathrm{Hz}$, its functional significance may be different $(5,13,14,38,39)$. In some subjects (for example SV), the response at a given frequency of the EEG could occur when this represents a double or triple frequency of the stimulation, but not when this is the fundamental frequency. However, in the present study the visual stimulation was presented through very short stroboscopic flashes (as available in standard EEG equipment) rather than by sinusoidally varying light intensities, as used by many other investigators. As a result, the stimulus input to the visual system occurs simultaneously at the fundamental frequency, and also at higher harmonics of the stimulus frequency. This may at least in part be responsible for the relatively strong harmonic driving response and for the high correlation between the profiles for the fundamental and higher harmonics that were observed in the present study. With flash stimulation, it is difficult to determine if activity at higher harmonics is due to the stimulus input at these higher harmonics, or to the nonlinear characteristics of the EEG response.

Thus, the frequency profile of the EEG photic driving suggested in the present research has enabled us to reveal some additional characteristics of the bioelectrical ac- 
tivity and functional state of the brain, which were not apparent in the resting EEG. The profile extracts and integrates amplitude responses and their harmonics at all the IPS frequencies applied, allows their comparison, and may be treated as equivalent to a spectrum. It may serve as a complex characteristic of the individual EEG which presumably provides, in comparison with the resting EEG, a fuller representation of the set of potential oscillators. It also reflects a relatively more standardized functional state of the brain due to external stimulation, which has been considered to minimize the effect of uncontrolled variations in a current state (16). This is important in both experimental and diagnostic applications, and is expected to decrease dispersion of EEG parameters and make them more comparable between subjects.

\section{Acknowledgments}

The authors are grateful to Dr. A.M.F.M. de Sá and Prof. A.F.C. Infantosi for software and useful discussions, and to the anonymous reviewers for comments which have helped to improve the paper.

\section{References}

1. Takahashi T (1993). Activation methods. In: Niedermeyer E (Editor), Electroencephalography: Basic Principles, Clinical Applications, and Related Fields. Williams \& Wilkins, Baltimore, MD, USA.

2. Chatrian GE, Bergamini L, Dondey $M$, Klass DW, Lennox-Buchthal M \& Petersén I (1974). A glossary of terms most commonly used by clinical electroencephalographers. Electroencephalography and Clinical Neurophysiology, 37: 538-548.

3. Walter VJ \& Walter WG (1949). The central effects of rhythmic sensory stimulation. Electroencephalography and Clinical Neurophysiology, 1: 57-86.

4. Tyler CW, Apkarian $P \&$ Nakayama K (1978). Multiple spatial-frequency tuning of electrical responses from human visual cortex. Experimental Brain Research, 33: 535-550.

5. Danilova NN (1985). Functional States: Mechanisms and Diagnostics (in Russian). Moscow University Press, Moscow, Russia.

6. Fedotchev Al, Bondar AT \& Konovalov VF (1990). Stability of resonance EEG reactions to flickering light in humans. International J ournal of Psychophysiology, 9: 189-193.

7. Basar E, Basar-Eroglu C, Rahn E \& Schurmann M (1991). Sensory and cognitive components of brain resonance responses. Acta Oto-Laryngologica, 491 (Suppl): 25-34.

8. Basar-Eroglu C, Basar E, Demiralp T \& Schurmann M (1992). P300-response: possible psychophysiological correlates in delta and theta channels. International
J oumal of Psychophysiology, 13: 161-179.

9. Sakamoto $H$, Inouye $T \&$ Shinosaki $K$ (1993). Presenvation of alpha rhythm shortly after photic driving. International J ournal of Neuroscience, 73: 227-233.

10. J in Y, Castellanos A, Solis ER \& Potkin SG (2000). EEG resonant responses in schizophrenia: a photic driving study with improved harmonic resolution. Schizophrenia Research, 44: 213-220.

11. Livanov MN (1977). Spatial Organization of Cerebral Processes. Wiley-Liss, New York, NY, USA.

12. Gevins A (1993). High resolution EEG. Brain Topography, 5: 321-325.

13. Takahashi $T$, Kataoka $K \&$ Tsukahara $Y$ (1988). Power spectral analysis of photic driving elicited by flickering dot pattern and red flicker stimuli in adult psychiatric outpatients - with special reference to age and gender. Tohoku J ournal of Experimental Medicine, 156: 165-173.

14. Drake J r ME, Shy KE \& Liss L (1989). Quantitation of photic driving in dementia with normal EEG. Clinical Electroencephalography, 20: 153-155.

15. Gronseth GS \& Greenberg MK (1995). The utility of the electroencephalogram in the evaluation of patients presenting with headache: a review of the literature. Neurology, 45: 1263-1267.

16. Kaiser J \& Gruzelier J H (1996). Timing of puberty and EEG coherence during photic stimulation. Intemational J oumal of Psychophysiology, 21: 135-149.

17. Petrenko ET (1988). Electroencephalographic changes in response to the equilibrium test and rhythmic light flashes (in
Russian). Kosmicheskaya Biologiya i Aviakosmicheskaya Meditsina, 22: 21-25.

18. Morse DR (1993). Brain wave synchronizers: a review of their stress reduction effects and clinical studies assessed by questionnaire, galvanic skin resistance, pulse rate, saliva, and EEG. Stress Medicine, 9: 111-126.

19. Fedotchev Al (1997). Analysis of EEG resonance reactions in evaluation of the effectiveness of sensory stimulation. Human Physiology (Fiziologiya Cheloveka), 23: 117-123.

20. Fox PT \& Raichle ME (1984). Stimulus rate dependence of regional cerebral blood flow in human striate cortex, demonstrated by positron emission tomography. J ournal of Neurophysiology, 51: 1109-1120.

21. Golding J F \& Richards M (1985). EEG spectral analysis, visual evoked potential and photic-driving correlates of personality and memory. Personality and Individual Differences, 6: 67-76.

22. Eeg-Olofsson O (1980). Longitudinal developmental course of electrical activity of brain. Brain and Development, 2: 3344.

23. Coull BM \& Pedley TA (1978). Intermittent photic stimulation. Clinical usefulness of non-convulsive responses. Electroencephalography and Clinical Neurophysiology, 44: 353-363.

24. Scheuler W (1983). Clinical significance of increased reaction to photostimulation in the alpha frequency range (in German). EEG-EMG Zeitschrift für Elektroenzephalographie, Elektromyographie und Ver- 
wandte Gebiete, 14: 143-153.

25. de Falco FA, Roberti R, Florio C \& Franzese $G$ (1992). Photoparoxysmal response on eye closure in photosensitive patients. Acta Neurologica, 14: 290-296.

26. Wolf $P \&$ Goosses R (1986). Relation of photosensitivity to epileptic syndromes. J ournal of Neurology, Neurosurgery, and Psychiatry, 49: 1386-1391.

27. Valmier J, Touchon J \& Baldy-Moulinier M (1989). Interictal regional cerebral blood flow during nonspecific activation test in partial epilepsy. J ournal of Neurology, Neurosurgery, and Psychiatry, 52: 364371.

28. Kooi KA, Tucker RP \& Marshall RE (1978). Fundamentals of Electroencephalography. Harper \& Row, Hagerstown, MD, USA.

29. Bickford RG (1979). Activation procedures and special electrodes. In: Klass DW \& Daly DD (Editors), Current Practice of Clinical Electroencephalography. Raven Press, New York, NY, USA, 269-305.

30. Beydoun A, Schechter SH, Nasreddine W \& Drury I (1998). Responses to photic stimulation in patients with occipital spikes. Electroencephalography and Clini- cal Neurophysiology, 107: 13-17.

31. de Tommaso M, Sciruicchio V, Bellotti R, Guido M, Sasanelli G, Specchio LM \& Puca $F$ (1999). Photic driving response in primary headache: diagnostic value tested by discriminant analysis and artificial neural network classifiers. Italian J oumal of Neurological Sciences, 20: 23-28.

32. Wada $\mathrm{Y}$, Takizawa $\mathrm{Y} \&$ Yamaguchi $\mathrm{N}$ (1995). Abnormal photic driving responses in never-medicated schizophrenia patients. Schizophrenia Bulletin, 21: 111-115.

33. Takahashi T \& Kataoka K (1990). Influence of indeloxazine hydrochloride upon photic driving responses elicited by flickering dot pattern and red flicker stimuli in elderly patients. J apanese J ournal of Psychiatry and Neurology, 44: 709-715.

34. J in Y, Potkin SG \& Sandman C (1995). Clozapine increases EEG photic driving in clinical responders. Schizophrenia Bulletin, 21: 263-268.

35. Danilova NN (1961). Reaction of cerebral electrical activity to light flickering which coincides with frequency band of alpha rhythm (in Russian). Zhurnal Vysshey Nervnoy Deyatel'nosti Imeni I.P. Pavlova,
11: 12-21.

36. Ilyanok VA (1969). Influence of the frequency of rhythmic photic stimulation on the harmonic composition of the driving response in the human brain. Electroencephalography and Clinical Neurophysiology, 27: 325.

37. Yaguchi K \& Iwahara S (1976). Temporal sequence of frequency specific and nonspecific effects of flickering lights upon the occipital electrical activity in man. Brain Research, 107: 27-38.

38. Hamel B, Bourne J R, Ward J W \& Teschan PE (1978). Quantitative assessment of photic driving in renal failure. Electroencephalography and Clinical Neurophysiology, 45: 719-730.

39. Nyrke T \& Lang AH (1982). Spectral analysis of visual potentials evoked by sine wave modulated light in migraine. Electroencephalography and Clinical Neurophysiology, 53: 436-442.

40. Gebber GL, Zhong S, Lewis C \& Barman SM (1999). Human brain alpha rhythm: nonlinear oscillation or filtered noise? Brain Research, 818: 556-560. 